\title{
Serial MRI studies over 12 months using manual and atlas-based region of interest in patients with amyotrophic lateral sclerosis
}

Ashwag R. Alruwaili ${ }^{1,2,3^{*}}$ D, Kerstin Pannek ${ }^{4}$, Robert D. Henderson ${ }^{3,5}$, Marcus Gray ${ }^{6,7}$, Nyoman D. Kurniawan ${ }^{6}$ and Pamela A. McCombe

\begin{abstract}
Background: Amyotrophic lateral sclerosis (ALS) is a neurodegenerative disease characterized by loss of upper and lower motor neurons. There is a need for an imaging biomarker to track disease progression. Previously, magnetic resonance imaging (MRI) has shown loss of grey and white matter in the brain of patients with ALS compared to controls. We performed serial diffusion tractography imaging (DTI) study of patients with ALS looking for changes over time.
\end{abstract}

Methods: On all subjects $(n=15)$, we performed three MRI studies at 6 month intervals. DTI changes were assessed with tract-based spatial statistics (TBSS) and region of interest (ROI) studies. Cortic-spinal tract (CST) was selected for our ROI at the upper level; the posterior limb of internal capsule (PLIC), and a lower level in the pons.

Results: There was no significant change in DTI measures over 12 months of observation. Better correlation of manual and atlas-based ROI methods was found in the posterior limb of the internal capsule than the pons.

Conclusion: While previous DTI studies showed significant differences between ALS subjects and controls, within individual subjects there is little evidence of progression over 12 months. This suggests that DTI is not a suitable biomarker to assess disease progression in ALS.

Keywords: Amyotrophic lateral sclerosis, ALS, Motor neuron disease, MND, Diffusion tensor imaging, DTI, Region of interest, ROI, Tract-based spatial statistics, TBSS

\footnotetext{
* Correspondence: ashalruwaili@ksu.edu.sa

${ }^{1}$ The University of Queensland, Centre for Clinical Research, Brisbane,

Australia

${ }^{2}$ King Saud University, Riyadh, Saudi Arabia

Full list of author information is available at the end of the article
}

(c) The Author(s). 2020 Open Access This article is licensed under a Creative Commons Attribution 4.0 International License, which permits use, sharing, adaptation, distribution and reproduction in any medium or format, as long as you give appropriate credit to the original author(s) and the source, provide a link to the Creative Commons licence, and indicate if changes were made. The images or other third party material in this article are included in the article's Creative Commons licence, unless indicated otherwise in a credit line to the material. If material is not included in the article's Creative Commons licence and your intended use is not permitted by statutory regulation or exceeds the permitted use, you will need to obtain permission directly from the copyright holder. To view a copy of this licence, visit http://creativecommons.org/licenses/by/4.0/ The Creative Commons Public Domain Dedication waiver (http://creativecommons.org/publicdomain/zero/1.0/) applies to the data made available in this article, unless otherwise stated in a credit line to the data. 


\section{Background}

Amyotrophic lateral sclerosis (ALS) is characterized by the progressive loss of lower motor neurons (LMN) from the spinal cord and brain stem and upper motor neurons (UMN) from the motor and premotor cortex. There can also be extra-motor involvement [1]. The pathology of ALS includes aggregation of protein within cells [2]. The protein accumulation spreads to other regions as disease progresses [3]. There is a need for non-invasive biomarkers as a means of monitoring progression of patients with ALS, for prognosis and for use in clinical trials. Possible biomarkers include clinical, neurophysiological, blood and imaging measurements. An imaging biomarker is desirable because it can show changes in brain structure that can be related to the pathology of disease.

Magnetic resonance imaging (MRI) has an advantage of being non-invasive, and able to quantify brain structure and to make inferences about tissue pathology. Cross sectional MRI studies in ALS have shown abnormalities in voxel-based morphometry (VBM) and diffusion weighted imaging (DWI) [4-7]. Because ALS is a disease that shows degeneration of white matter fiber tracts, particularly the corticospinal tract (CST), there have been studies that use diffusion tensor imaging (DTI). DTI can be analyzed with Tract-Based Spatial Statistics (TBSS) [8] or region of interest (ROI) studies, which can be can be performed by manual or semi-automated methods. Manually drawn ROIs have been considered to have problems with reproducibility, but have the advantage of ease of use.

All DTI metrics have been found to reflect microstructural pathology. What we know about DTI metrics is largely based upon empirical studies that investigate how these measures correlate with the underlying pathology. Quantitative FA measures are believed to reflect changes in the myelination, fiber density and packing [9]. Evidence suggests that fractional anisotropy (FA) is sensitive to the WM integrity but is not very specific and cannot distinguish between different pathological processes [10-12]. Mean diffusivity (MD) is suggested to be influenced by free water diffusion in cellular space, such as oedema, which is increased in neurodegenerative diseases [12]. Axial diffusivity (AD) is thought to reflect demyelination in white matter tracts. Axial integrity of axons and can be reflected as either increased or decreased $\mathrm{AD}$ when WM is destroyed by any other process [13]. It has not yet been well-defined whether radial diffusivity (RD) is specific to demyelination and could also be related to be due to; the occurrence of inflammation, or to axonal damage. RD was found to be high in neurodegenerative disease [14]. RD has been shown to correlate with demyelination and dysmyelination in several pathological models $[15,16]$.

Cross-sectional DTI studies have consistently shown reduction in FA in the corticospinal tract (CST) in ALS patients compared to controls [17-20]. In contrast, longitudinal DTI studies of ALS have shown conflicting findings, but most studies show DTI abnormalities early in disease, but little progression over time [7, 21-26].

We have previously reported a study comparing scans 6 months apart, and found little difference in DTI measures [24]. In this study we have focused on the CST, because degeneration of the CST is a cardinal feature of the pathology in ALS. To examine whether changes can be detected after a longer interval, we now report a 12 month serial study of patients with ALS, with the aim of determining whether there are changes over time and the second aim was to compare semi-automated ROI with manual ROI methods.

\section{Methods \\ Subjects}

Patients $(n=15)$ with clinically definite or clinically probable ALS, according to the revised El Escorial criteria [27] were recruited from the motor neuron disease (MND) clinic of the Royal Brisbane and Women's Hospital (RBWH) and were included in previous studies [20, 24]. They had MRI imaging three times at 6 monthly intervals. Disease severity was measured using the revised ALS Functional Rating Scale (ALSFRS-R) [28]. This project was approved by the RBWH ethics committee HREC (2008/98). All subjects provided written informed consent.

\section{MRI acquisition}

All MRI scans were performed at the Royal Brisbane and Women Hospital (RBWH) using a $3 \mathrm{~T}$ Siemens Tim Trio (Siemens, Erlangen, Germany) with a 12-channel parallel head coil. Diffusion-weighted images (DWIs) were acquired along 64 non-collinear directions at $\mathrm{b}=3000 \mathrm{~s} / \mathrm{mm}^{2}$, with one non-diffusion weighted image. Acquisition parameters were: 60 axial slices, FOV $30 \times 30 \mathrm{~cm}$, slice thickness 2.5 mm, matrix $128 \times 128$, TR/TE 9700/119 ms, iPAT factor 2 . A field map was acquired using two $2 \mathrm{D}$ gradient-recalled echo images with TE1/TE2 $=4.92 / 7.38 \mathrm{~ms}$ and $\mathrm{TR}=488$ $\mathrm{ms}$ to assist in the correction of geometric distortions. The acquisition time for the diffusion dataset was 9:40 min.

In addition, a high-resolution structural image was acquired using a $3 \mathrm{D} T_{1}$ MPRAGE (FOV $23 \mathrm{~cm}$ slice thickness $0.9,0.44 \times 0.44 \mathrm{~mm}$ in-plane resolution, matrix $24 \times 25.6 \times$ $17.6 \mathrm{~cm}, \mathrm{TR} / \mathrm{TE} / \mathrm{TI} 1900 / 2.4 / 900 \mathrm{~ms}$, flip angle $=9$ degrees $)$.

\section{Diffusion processing}

Diffusion MRI data were preprocessed as described previously [29]. Preprocessing included correction for head movement with rotation of the b-matrix, detection and removal of signal intensity outliers, and correction for geometric distortions and intensity inhomogeneity. Maps of FA were calculated using MRTrix version 0.2.9 [30]. 


\section{TBSS analysis}

Tract-based spatial statistics (TBSS) is a tool for analyzing DTI variables, which allows objective voxel-wise analysis. TBSS provides a powerful method for detecting variation on major white matter tract. The alignment of the DT images to a reference space is required. This allows spatially overlapping voxels of different datasets to correspond to the same anatomical structures.

Following registration, the mean FA-image was created and thinned to represent the mean FA skeleton. Individual FA volumes were projected onto this common skeleton [31]. Next, data were fed into voxel-wise cross-subject statistical analysis with different group comparisons, including a paired test. The statistical analyses employed the voxelwise general linear model (GLM) and significant clusters were formed by employing the TFCE method to correct for multiple comparisons [32], implemented in FSL randomize [33]. P-values were determined using 5000 random permutations [33]. The results are considered significant at $p<$ 0.05 , corrected for multiple comparisons using TFCE.

To analyze differences among three groups, we combined all the scans and ran the TBSS steps as above to create FA skeleton of whole group. Before the randomise step, we separated scans for each time-point from the FA skeleton. Next, two time-points were merged to one skeleton. Then, using randomise with multiple permutations and correcting for multiple comparisons using threshold-free cluster enhancement (TFCE, [32]), which is an approach to avoid the problem of thresholding selection in the context of cluster-based statistics. At $p<0.05$, we ran two different statistical approaches: (a) one sample t-test; by subtracting each time-point from the other one in six contrasts (1 minus 2, 1 minus 3, 2 minus 1,2 minus 3, 3 minus 1, 3 minus 2) and the output is the difference between time-points; (b) paired sample t-test; comparing two time-points each time, separately, using the contrast as (scan 1 versus scan 2, scan 2 versus scan 3 , scan 1 versus scan 3); c) triple t-test; comparing all three timepoints. The final results from TBSS comparisons of ALS patients at each time-point were superimposed using FSL (http://fsl.fmrib.ox.ac.uk/fsl/fslwiki/).

\section{Automated ROI analysis methods}

For the automated ROI, Advanced Normalization Tools (ANTs) was performed on FA data using the methods described [24]. A custom FA template was derived from all subjects, using scripts provided with ANTs (http://picsl. upenn.edu/ANTS/) [34]. We used ANTs symmetric diffeomeophic registrations using symmetric image normalization (Greedy SyN). The JHU 1-mm FA was used for the initial rigid body registration to generate the template.

The JHU atlas [35] was normalized to this study template using symmetric diffeomorphic registration. ROIs of the JHU atlas were subsequently transformed to the individual datasets in native space and mean values of FA were calculated for each ROI.

The datasets were analysed by one blinded rater (A, $\mathrm{R}$ A). Manual ROI were drawn using FSL (http:// www.fmrib.ox.ac.uk/fsl/) on the basis of the prior anatomic knowledge and relevant literature and saved as a mask [36].

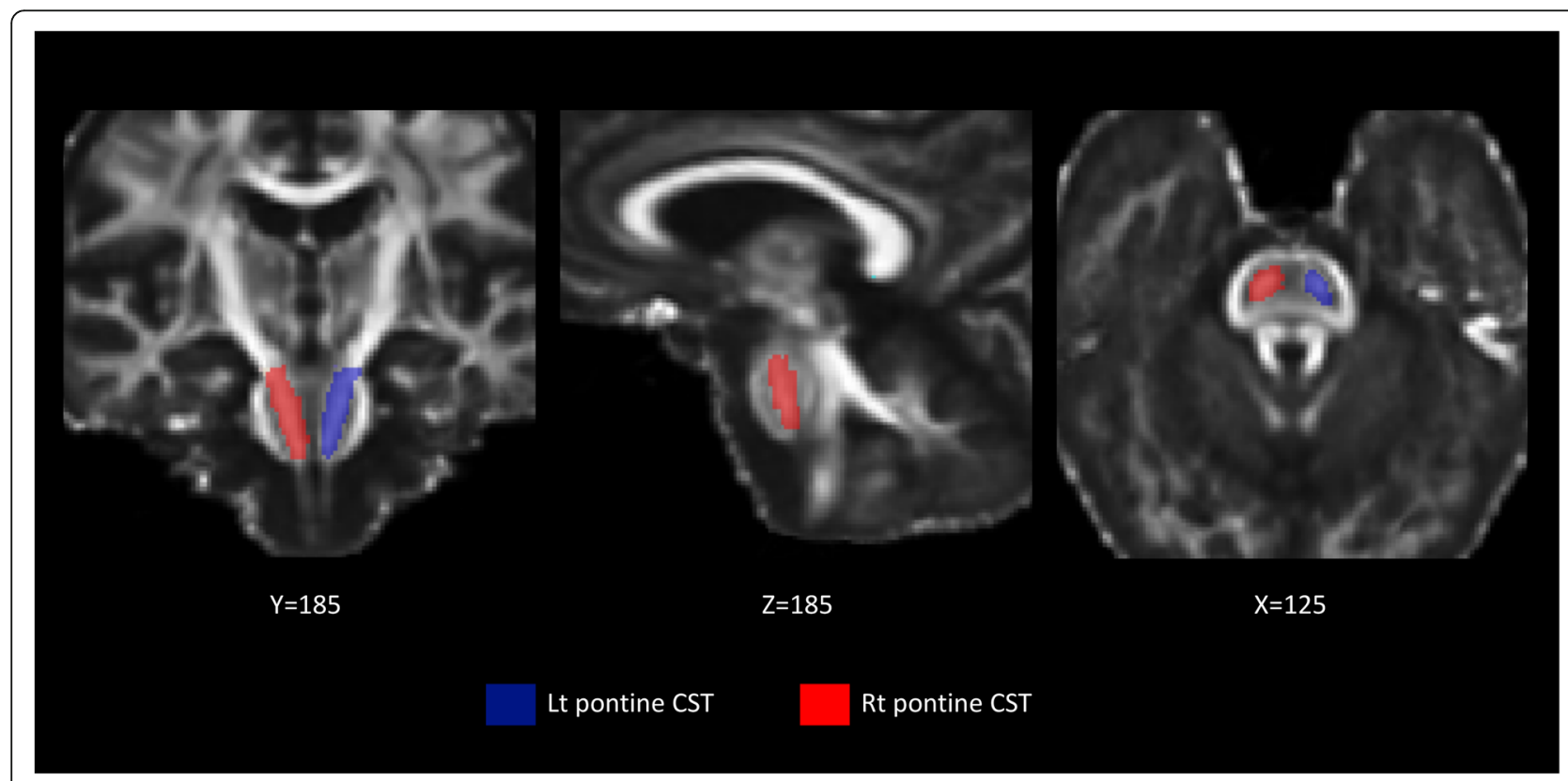

Fig. 1 Atlas-based ROI of the CST at the pons. This Figure illustrates, in 3 planes ( $X, Y$ and Z), the mask applied for the CST in the pons using the FA template with atlas-based ROI. (Left in blue and right in red). With this method, all the voxels from the entire structure are included in the ROI 


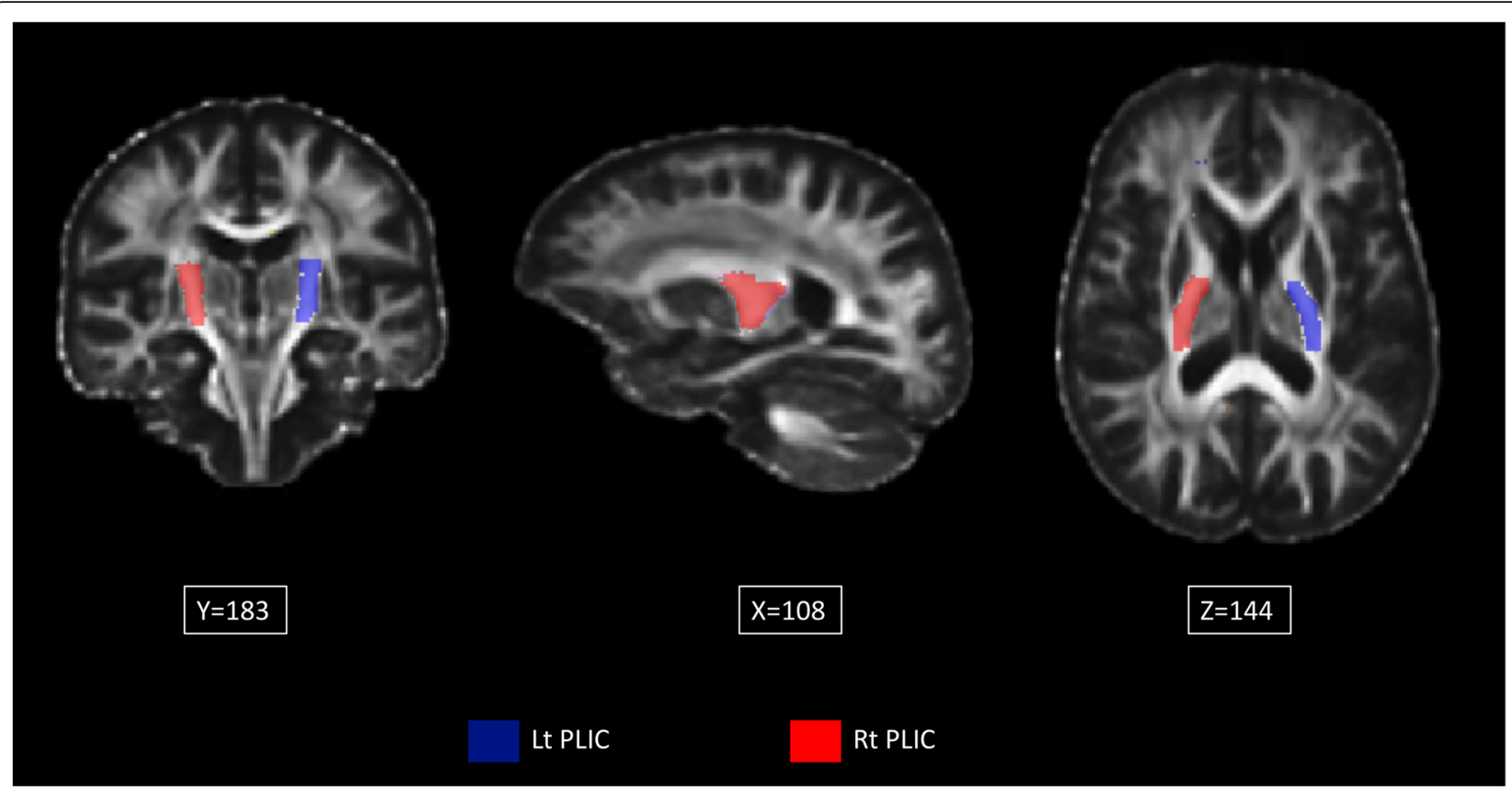

Fig. 2 Atlas-based ROI of the PLIC. This Figure illustrates, in 3 planes ( $X, Y$ and $Z$ ), the mask applied for the PLIC, using the FA template with atlasbased ROI. (left in blue and right in red). With this method, all the voxels from the entire structure are included in the ROI

We examined the CST at the level of pons as shown in Fig. 1 and the posterior limb of the internal capsule (PLIC) as shown in Fig. 2. These regions were chosen because degeneration of the CST is a cardinal feature of ALS. PLIC and pons are part of the CST where PLIC is upper and pons is lower CST. We included the PLIC, the nearest CST to Gray matter (GM), expecting the highest sensitivity where highest neuronal alignment and no crossing fibers present. The FA values were calculated pixel-by-pixel, and average values for each region on both sides were tabulated for statistical analysis.

\section{Manual ROI methods}

Using knowledge of anatomy, manual ROIs were drawn on the individual colored FA maps. One axial slice was selected for each region. To delineate the CST in the pons, the ROI was drawn where the pontine crossing tracts (pct) are a straight red line. The pontine CST can be seen as two blue-purple regions anterior to the pct (Fig. 3, left). For the PLIC, the ROI was drawn on the last axial slice where the genu and splenium of corpus callosum and the fornix are seen in one plane. On

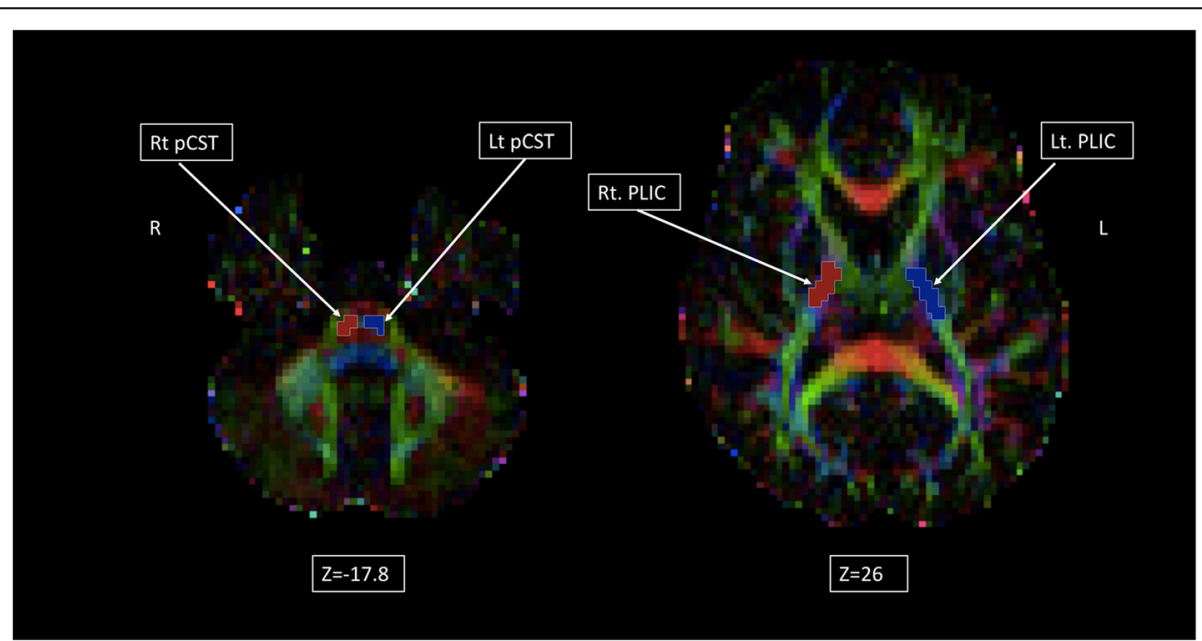

Fig. 3 Manual ROI of the CST at the pons and PLIC. This Figure illustrates in two different axial planes for individual patient FA maps and the mask applied for the manual ROI for the pontine CST (left) and posterior limb of internal capsule, PLIC (right). With this method, only the voxels from one plane are included in the mask of the ROI 
colored FA maps, the PLIC appears as a blue-purple region; anterior to it is the anterior limb of internal capsule (ALIC) and posterior to the PLIC is the retrolenticular part of the internal capsule (rLIC), (Fig. 3, right). To assess repeatability of ROIs, five patients were randomly selected to have a repeat of the manual ROI, with the repeat analysis done a minimum of 1 month after the first analysis.

\section{Statistical analysis}

Statistical analyses were performed using SPSS for Mac (Ver. 23.0, SPSS Inc., Chicago, IL, USA). Mean and standard deviation values were calculated for each variable. All data were tested for normality using ShapiroWilk test. MANOVA was performed when testing ROIs at three time-points for each region of interest. We pooled all the FA data from all subjects from all timepoints to examine the overall trend of the relationship between both ROI methods. We examined the relationship between manual ROI and atlas ROI using Spearman's correlation. Repeatability of the manual ROI was calculated using within-subject coefficient of variation $(\mathrm{CV})$ and the intra-class correlation coefficient (ICC).

\section{Results}

\section{Subjects}

Fifteen ALS patients (mean age $60 \pm 13,10$ males and 5 females) were enrolled (Tables 1 and 2). The subjects had a disease duration (DD), that was calculated from the date of onset to the first MRI scan, and ranged from 3 to 118 months. Physical decline in ALSFRS-R are available in Table 3.

\section{TBSS results}

The detailed results of TBSS analyses are shown in Table 4. There was no significant difference among the scans of ALS subjects at three time-points when comparing whole ALS group or when excluding patients with longer disease duration more than 40 months $(n=$ $3, p<0.05$; corrected TFCE). The changes in the CST and corpus callosum (CC) were more widespread at the third scans than in the earlier time-points.

Table 1 Summary of subjects demographics

\begin{tabular}{ll}
\hline & ALS \\
& $N=15$ \\
\hline Age (years) Mean (SD) & $60 \pm 13^{*}$ \\
Gender (Male:female) & $10: 5^{\mathrm{a}}$ \\
ALSFRS-R Mean (SD) & $38.7 \pm 4.5$ \\
Disease duration Mean (SD) Median (months) & $35 \pm 2729$ \\
Range of disease duration (months) & $3-118$ \\
Number of patients deceased at end of study & 6 \\
\hline
\end{tabular}

${ }^{*} p=0.005 ;{ }^{a}$ not significant

\section{ROI results}

With both atlas-based and with manual ROI analysis of FA, there was considerable variation among individuals (Supp Figures 1 and 2). We performed MANOVA to determine whether the average FA values were significantly different over time (Supp Table 1). There was no significant difference among the three time-points for either method. Therefore, we acquired the estimated marginal means for each time-point using both methods to test whether both methods are comparable (Supp Figure 3). There was better agreement for the PLIC than for the CST. A summary of the FA results in all ROIs is shown in Fig. 4.

To compare the FA value between the PLIC and pontine CST, we have combined the $90 \mathrm{FA}$ values for both sides from all time-points and calculated the mean for two methods (Supp Table 2). The average FA from the PLIC is significantly greater than that of the CST in the pons, using both methods.

A linear regression was performed between atlas-based and manual-based measurements for each ROI using all the observations from the three time-points (Fig. 5). There was better agreement for the values from the PLIC than the values from the pons. We also performed a Spearman's correlation of ROI measurements to compare the manual versus atlas-based ROI methods testing if both methods are comparable (Supp Table 3). The correlation in the left PLIC was significant $(p=0.01, r=0.6)$.

\section{Reproducibility of manual ROI}

Reproducibility of manual ROI was tested with 1 month difference between manual ROI placements. The withinsubject CV was used to test repeatability of both ROI methods. In the right PLIC, CV was $0.1-3.6 \%$ in the aROI and was $0.007-11 \%$ in the mROI.

In terms of reliability, the inter-rater and intra-rater variability can be assessed using the ICC [37]. This have been reported to be between 17 and 37\% [38]. In our study, ICC was between 24 and $38 \%$ for the right pons while the left pons was $74-85 \%$. In the right PLIC, the ICC was $37-54 \%$ and the left PLIC was $65-79 \%$.

\section{Discussion}

This study shows that there is no significant change in DTI along CST in the selected ROIs in ALS patients over 12 months. Manual and automated ROI methods gave comparable results.

The underlying pathology of ALS is death of upper motor neurons, degeneration of the CST, and death of lower motor neurons, with eventual gliosis of the CST in the spinal cord. In the present study, we have examined changes in DTI measures, with the hypothesis that there would be degeneration over time seen as loss of FA in WM tracts. We used TBSS for an overview of all the fiber tracts and ROI of the PLIC and CST to determine 
Table 2 Detailed clinical information of ALS subjects

\begin{tabular}{llllllll}
\hline & ID & Familial/Sporadic & Handedness & RILUZOLE & Site of Onset & Date of Onset & Disease duration \\
\hline 1 & 303 & S & Right & N & LUL & $01 / 05 / 2010$ & 33 \\
2 & 304 & S & Right & N & RUL & $01 / 08 / 2008$ & 54 \\
3 & 306 & S & Right & Y & RUL & $01 / 10 / 2010$ & 29 \\
4 & 308 & S & Right & N & LL (Bilateral) & $01 / 01 / 2008$ & 61 \\
5 & 311 & S & Right & Y & LUL & $01 / 06 / 2010$ & 34 \\
6 & 313 & S & Right & Y & RLL & $01 / 05 / 2011$ & 25 \\
7 & 315 & S & Right & Y & LUL & $01 / 03 / 2012$ & 15 \\
8 & 316 & F & Right & N & LUL & $01 / 08 / 2013$ & 3 \\
9 & 318 & S & Right & N & RUL & $01 / 08 / 2012$ & 14 \\
10 & 319 & S & Right & NA & LLL & $01 / 09 / 2010$ & 37 \\
11 & 320 & S & Right & N & RUL & $01 / 01 / 2004$ & 118 \\
12 & 324 & S & Right & N & LLL & $01 / 07 / 2012$ & 20 \\
13 & 325 & S & Right & N & Bulbar & $01 / 09 / 2011$ & 31 \\
14 & 329 & S & Left & N & LLL & $01 / 02 / 2012$ & 29 \\
15 & 333 & S & Right & N & Bulbar & $01 / 07 / 2013$ & 23
\end{tabular}

whether MRI can show changes in these motor pathways. We find that there is no significant change in DTI over a 12 month period. We also find that the PLIC shows better correlation between manual and automated ROI measures than pons.

Longitudinal DTI studies in ALS are challenging and the findings are variable, but generally concur that fiber tract degeneration has typically taken place by the time the diagnosis is confirmed, with limited additional WM changes in later phase of the disease [39]. An older longitudinal DTI study with only six noncollinear directions

Table 3 ALS subjects' physical decline

\begin{tabular}{lll}
\hline Subject ID & First ALSFRS-R & Last ALSFRS-R \\
\hline 1 & 42 & 34 \\
2 & 47 & 45 \\
3 & 37 & 30 \\
4 & 33 & 33 \\
5 & 35 & 42 \\
6 & NA & NA \\
7 & 31 & 29 \\
8 & NA & NA \\
9 & 38 & 30 \\
10 & 34 & NA \\
11 & 48 & 42 \\
12 & 41 & 40 \\
13 & 46 & 41 \\
14 & 43 & 42 \\
15 & 44 & NA \\
\hline
\end{tabular}

have reported positive findings but on a very small sample size [40]. Our own previous study found little change in DTI over 6 months [24]. In the present study we examined changes over a 12 month period, to see whether DTI changes appear over a longer time interval.

Data were investigated at three different time-points, 6 months apart. With TBSS there was some evidence of change over time in motor pathways. The ROI analyses were used to test our hypothesis that there would be changes in the FA in the CST at pons and PLIC. There were no significant differences in the average FA between time-point using aROI. However, in this study some patients showed loss of FA over time while others did not. This difference between TBSS and ROI has been noted previously, in a study that found ROI methods to show less significant results than TBSS methods [41].

There are some reasons why there would be little change in FA over time. Firstly, ALS patients are heterogeneous, with some patients showing prolonged survival [42-44]. In our cohort, some of the patients had prolonged survival, indicating slowly progressive disease, and therefore would not be expected to show rapid change in fiber tracts. It may be that patients with rapidly progressing disease would show DTI changes.

Next, it is likely that after a fiber tract has completely degenerated, there will be no further change in DTI. Some of our patients were in later stages of the disease, when fiber tracts could be completely degenerated. If loss of fiber tracts leads to decline in FA, then the decline would be expected to cease at this point.

However, it is also possible that, even early in disease, the degeneration in the motor tracts is substantially advanced. Other studies have reported little change in DTI 
Table 4 Results of comparisons that were examined with TBSS. All group comparisons were not significant

\begin{tabular}{llll}
\hline Comparison of 3 groups & & & \\
\hline Group 1 & Group 2 & Group 3 & Significance \\
\hline Time-point 1 $(N=15)$ & Time-point 2 $(N=15)$ & Time-point 3(N=15) & NS \\
Time-point 1 exclude longer DD $(N=12)$ & Time-point 2 excluding longer DD $(N=12)$ & Time-point 3 excluding longer DD (N=12) & NS
\end{tabular}

DD Disease duration, NS Not significant

over time $[7,45]$. These authors suggested that this indicates that upper motor neuron degeneration occurs early in the disease. Our previous MRI study showed that UMN degeneration occurs early [46], and neurophysiological studies also suggest this [47]. This could mean that changes in FA occur early in disease. It may be that it is better to measure spread of DTI changes to new regions rather than follow changes in the CST [48]. Heterogeneity of clinical features in ALS patients is also an issue and subgroup analysis according to features such as the presence of cognitive impairment would be useful.

In the current study we compared atlas based and manual ROI. The manual methods gave a wider spread of values of FA. We found better agreement from the PLIC than the CST in the pons. Linear regression showed a significant relationship between the manual and atlas based methods for the PLIC but not the pons. This can be explained by the crossing WM pathways in

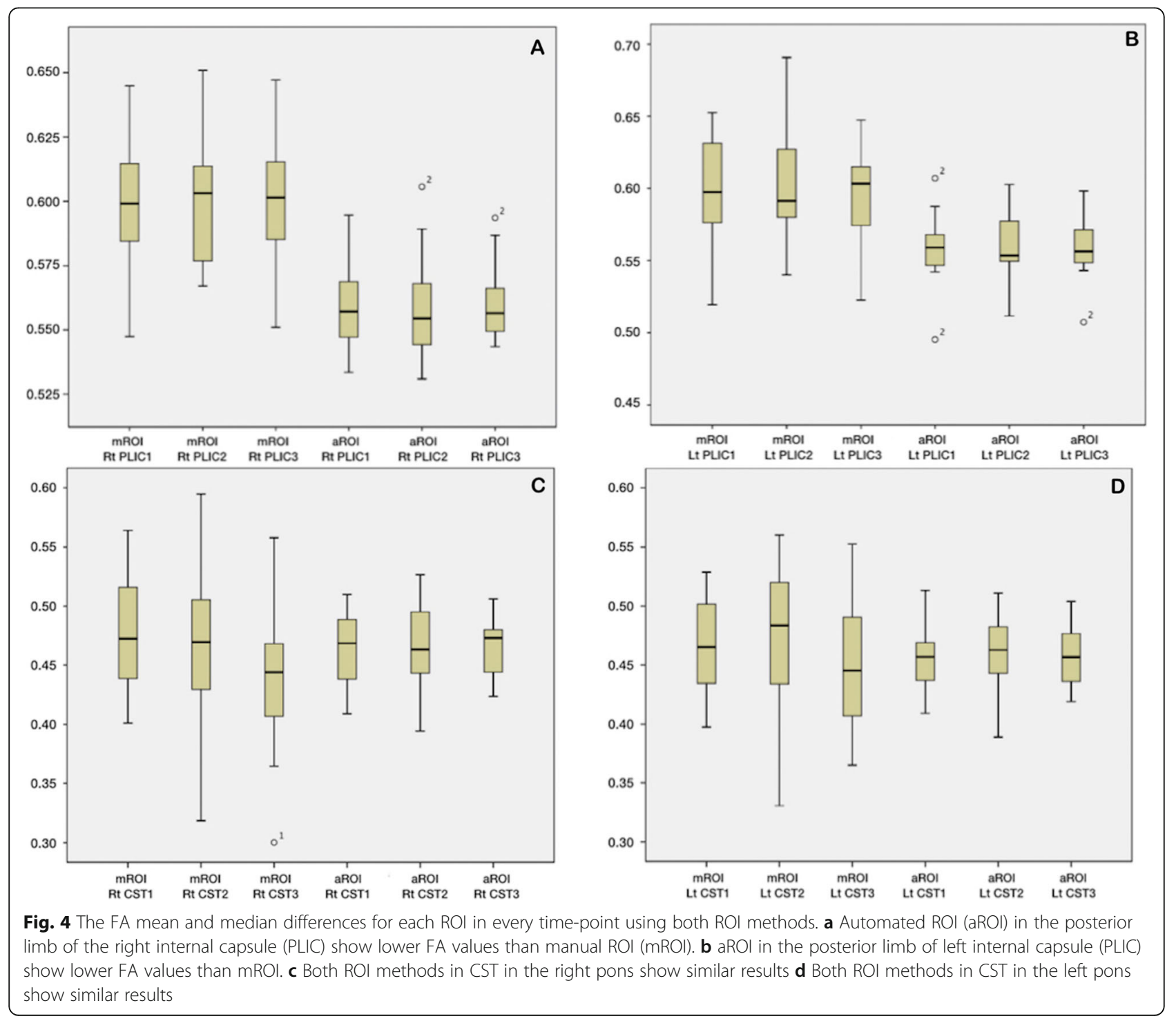



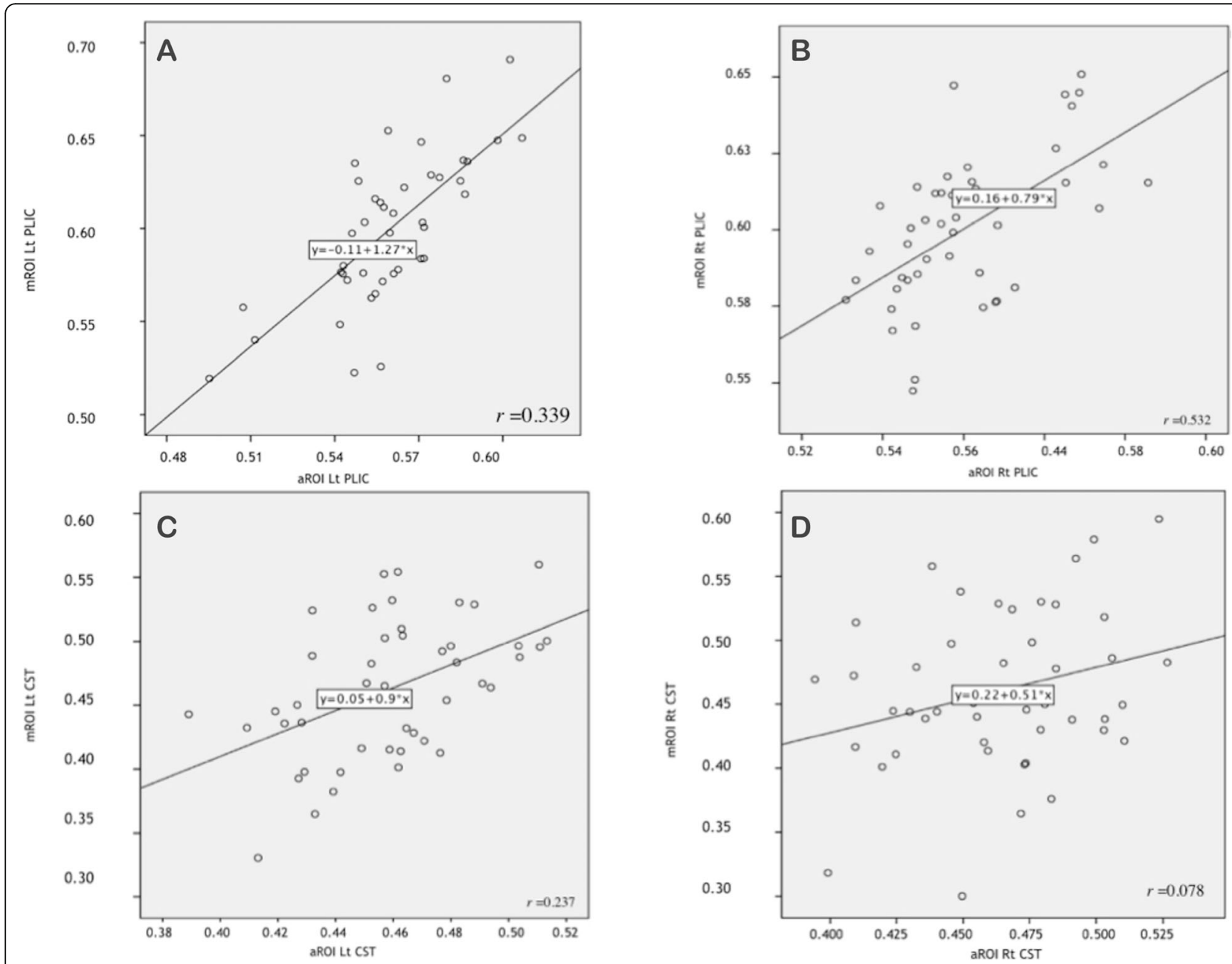

Fig. 5 Regression results for FA values for all scans in every ROI comparing manual ROI and automated ROI. a Regression analysis of mROI versus aROI for the left PLIC. $\mathbf{b}$ Regression analysis of $\mathrm{mROI}$ versus aROI for the right PLIC. c Regression analysis of mROI versus aROI for the left CST in the pons. $\mathbf{d}$ Regression analysis of $\mathrm{mROI}$ versus $\mathrm{aROI}$ for the right CST in the pons

the pons which can lead to a substantial drop of FA in the map. Using Spearman's correlation, there was a significant correlation of the values for the left PLIC. Manual ROI has been criticized for being operator dependent [49]. However, we found that both methods are still comparable which is in support of previous studies [50, 51]. Rigorous published work have compared different automated methods [52] and test-retest reproducibility [53] in segmentation approaches suggests that atlas-based methods are the best [54].

Laterality was not the aim in the current study. However, it is possible that our lack of significant difference in pons was due to not only the presence of crossing fibers but also to the challenges in delineating this structure, due to its small size, and difficulty discerning its boundaries within the brain stem. However, this variability in pons does limit the interpretation of our findings.

Previous studies have reported that although inter-site DTI measurement variability was found to be quite high
[55], the intra-subject variation was less than $1 \%$ in different brain regions using DTI [56]. Our patients showed variation of more than $1 \%$, therefore changes we observed are unlikely to be caused by measurement error alone. Compared to previous studies, the ICC from the current study shows that the manual ROI has a good reproducibility and the regions were drawn consistently [57].

Limitations of our study include the small sample size. More investigations are needed to characterize different ALS stages with a larger group of cohorts.

\section{Conclusion}

In conclusion, we found little evidence of progression of ALS using DTI. It may be that there is little further change after diagnosis because the pathological changes are complete. This study adds more information about the use of DTI in ALS longitudinal studies. 


\section{Supplementary information}

Supplementary information accompanies this paper at https://doi.org/10. 1186/s12880-020-00489-w.

Additional file 1 Figure S1. Atlas-based ROI FA measurement for individual patients at three time- points in the PLIC and CST in the pons. Using MANOVA there were no significant difference among the mean values at each time-points.

Additional file 2 Figure S2. Manual ROI FA measurement for individual patients at three time-points for the CST at the pons and PLIC. Using MANOVA there were no significant difference among the mean values at each time-points.

Additional file $\mathbf{3}$ Figure S3. Estimated marginal means for every ROI in both methods ( $\mathrm{mROI}$ in blue (1) and $\mathrm{aROI}$ in green (2)). There was better agreement for the PLIC (upper panels) than for the CST in the pons (lower panels).

Additional file 4 Table S1. Means (SD) for FA at three Time-points using atlas-based ROI and manual ROI. None of the regions, PLIC and CST at pons, showed significant differences overtime.

Additional file $\mathbf{5}$ Table S2. FA averaged mean of ROls in both methods.

Additional file $\mathbf{6}$ Table S3. Correlation of FA in both methods. Left PLIC showed significant correlation using both ROI methods.

\section{Abbreviations}

ALS: Amyotrophic lateral sclerosis; ALSFRS-R: The revised ALS Functional Rating Scale; ALIC: Anterior limb of internal capsule; aROI: Automated Region of interest; AD: Axial diffusivity; CC: Corpus callosum; CST: Corticospinal tract; CV: Coefficient of variation; DTI: Diffusion tensor imaging; DWl: Diffusion weighted imaging; DD: Disease duration; FA: Fractional anisotropy; FOV: Field of view; GLM: General linear model; GM: Gray matter; ICC: Intraclass correlation coefficient; LL: Left limb; LMN: Lower motor neuron; LUL: Left upper limb; MD: Mean diffusivity; mROI: Manual region of interest; MND: Motor neuron disease; MRI: Magnetic resonance imaging; NS: Not significant; PLIC: Posterior limb of internal capsule; pct: Pontine crossing tracts; RD: Radial diffusivity; rLIC: Retrolenticular part of the internal capsule; TBSS: Tract-based spatial statistics; TE: Echo time; TFCE: Threshold-free cluster enhancement; TI: Inversion time; TR: Repetition time; UMN: Upper motor neuron; ROI: Region of interest; rLIC: Retrolenticular part of internal capsule; RUL: Right upper limb; VBM: Voxel-based morphometry; WM: White matter

\section{Acknowledgements}

The authors wish to acknowledge the contributions of the participants and also to thank the research nurses who assisted with the project.

\section{Authors' contributions}

ARA is the major contributor in writing the manuscript, analyzed and interpreted the imaging data. PM and KP contributed to reviewing the methodology and final manuscript. PM and $\mathrm{RH}$ were responsible for patient recruitment. MG and NK contributed to image processing. All authors read and approved the final manuscript.

\section{Funding}

This research project was part of Dr. Alruwaili's PhD which was supported by a grant from the "Research Center of the Female Scientific and Medical Colleges", Deanship of Scientific Research, King Saud University.

\section{Availability of data and materials}

The datasets generated and analysed during the current study are not publicly available but are available from the principal investigator Prof. Pamela McCombe pamela.mccombe@uq.edu.au on reasonable request.

\section{Ethics approval and consent to participate}

This project was approved by the Royal Brisbane and Women's Hospita (RBWH) Human Research Ethics Committees (HREC) (2008/98). All subjects provided written informed consent.

\section{Consent for publication}

Not applicable.

\section{Competing interests}

The authors declare that they have no competing interests.

\section{Author details}

${ }^{1}$ The University of Queensland, Centre for Clinical Research, Brisbane, Australia. ${ }^{2}$ King Saud University, Riyadh, Saudi Arabia. ${ }^{3}$ School of Medicine, The University of Queensland, Brisbane, QLD, Australia. ${ }^{4}$ Australian E-Health Research Centre, CSIRO, Brisbane, Australia. ${ }^{5}$ The Department of Neurology, Royal Brisbane and Women's Hospital, Herston, Brisbane, Australia. ${ }^{6}$ Centre for Advanced Imaging, The University of Queensland, Brisbane, Australia. ${ }^{7}$ Gehrmann Laboratory, University of Queensland, Brisbane, QLD, Australia.

Received: 13 November 2019 Accepted: 23 July 2020

Published online: 03 August 2020

\section{References}

1. McCombe PA, Wray NR, Henderson RD. Extra-motor abnormalities in amyotrophic lateral sclerosis: another layer of heterogeneity. Expert Rev Neurother. 2017;17(6):1-17. https://www.tandfonline.com/doi/abs/10.1 080/14737175.2017.1273772

2. Blokhuis AM, Groen EJ, Koppers $M$, van den Berg LH, Pasterkamp RJ. Protein aggregation in amyotrophic lateral sclerosis. Acta Neuropathol. 2013;125(6):777-94.

3. Brettschneider J, Del Tredici K, Toledo JB, Robinson JL, Irwin DJ, Grossman $\mathrm{M}$, et al. Stages of pTDP-43 pathology in amyotrophic lateral sclerosis. Ann Neurol. 2013;74(1):20-38

4. Graham JM, Papadakis N, Evans J, Widjaja E, Romanowski CA, Paley MN, et al. Diffusion tensor imaging for the assessment of upper motor neuron integrity in ALS. Neurology. 2004;63(11):2111-9.

5. Hong $\mathrm{YH}$, Lee KW, Sung JJ, Chang KH, Song IC. Diffusion tensor MRI as a diagnostic tool of upper motor neuron involvement in amyotrophic lateral sclerosis. J Neurol Sci. 2004;227(1):73-8.

6. Chang JL, Lomen-Hoerth C, Murphy J, Henry RG, Kramer JH, Miller BL, et al. A voxel-based morphometry study of patterns of brain atrophy in ALS and ALS/FTLD. Neurology. 2005;65(1):75-80.

7. Bede P, Hardiman O. Longitudinal structural changes in ALS: a three timepoint imaging study of white and gray matter degeneration. Amyotrop Lateral Scler Frontotemporal Degener. 2018;19(3-4):232-41.

8. Smith SM, Jenkinson M, Johansen-Berg H, Rueckert D, Nichols TE, Mackay CE, et al. Tract-based spatial statistics: voxelwise analysis of multi-subject diffusion data. Neurolmage. 2006;31(4):1487-505.

9. Mädler B, Drabycz SA, Kolind SH, Whittall KP, MacKay AL. Is diffusion anisotropy an accurate monitor of myelination?: correlation of multicomponent $\mathrm{T} 2$ relaxation and diffusion tensor anisotropy in human brain. Magn Reson Imaging. 2008;26(7):874-88.

10. Sbardella E, Tona F, Petsas N, Pantano P. DTI measurements in multiple sclerosis: evaluation of brain damage and clinical implications. Mult Scler Int. 2013;2013:671730

11. Assaf $Y$, Pasternak $O$. Diffusion tensor imaging (DTI)-based white matter mapping in brain research: a review. J Mol Neurosci. 2008;34(1):51-61.

12. Filippi M, lannucci G, Cercignani M, Assunta Rocca M, Pratesi A, Comi G. A quantitative study of water diffusion in multiple sclerosis lesions and normal-appearing white matter using echo-planar imaging. Arch Neurol. 2000;57(7):1017-21.

13. Mayer AR, Ling J, Mannell MV, Gasparovic C, Phillips JP, Doezema D, et al. A prospective diffusion tensor imaging study in mild traumatic brain injury. Neurology. 2010;74(8):643-50.

14. Sun S-W, Liang H-F, Trinkaus K, Cross AH, Armstrong RC, Song S-K. Noninvasive detection of cuprizone induced axonal damage and demyelination in the mouse corpus callosum. Magn Reson Med. 2006;55(2):302-8.

15. Song S-K, Sun S-W, Ramsbottom MJ, Chang C, Russell J, Cross AH. Dysmyelination revealed through MRI as increased radial (but unchanged axial) diffusion of water. Neurolmage. 2002;17(3):1429-36

16. Fink F, Klein J, Lanz M, Mitrovics T, Lentschig M, Hahn HK, et al. Comparison of diffusion tensor-based tractography and quantified brain atrophy for analyzing demyelination and axonal loss in MS. J Neuroimaging. 2010;20(4):334-44. 
17. Abe O, Yamada H, Masutani Y, Aoki S, Kunimatsu A, Yamasue H, et al. Amyotrophic lateral sclerosis: diffusion tensor tractography and voxel-based analysis. NMR Biomed. 2004;17(6):411-6.

18. Karlsborg M, Rosenbaum S, Wiegell M, Simonsen H, Larsson H, Werdelin L, et al. Corticospinal tract degeneration and possible pathogenesis in ALS evaluated by MR diffusion tensor imaging. Amyotroph Lateral Scler Other Motor Neuron Disord. 2004;5(3):136-40.

19. Sarro L, Agosta F, Canu E, Riva N, Prelle A, Copetti M, et al. Cognitive functions and white matter tract damage in amyotrophic lateral sclerosis: a diffusion tensor tractography study. AJNR Am J Neuroradiol. 2011;32(10):1866-72.

20. Alruwaili AR, Pannek K, Coulthard A, Henderson R, Kurniawan ND, McCombe P. A combined tract-based spatial statistics and voxel-based morphometry study of the first MRI scan after diagnosis of amyotrophic lateral sclerosis with subgroup analysis. J Neuroradiol. 2018;45:41-8.

21. Keil C, Prell T, Peschel T, Hartung V, Dengler R, Grosskreutz J. Longitudinal diffusion tensor imaging in amyotrophic lateral sclerosis. BMC Neurosci. 2012;13(1):141.

22. Nickerson JP, Koski CJ, Boyer AC, Burbank HN, Tandan R, Filippi CG. Linear longitudinal decline in fractional anisotropy in patients with amyotrophic lateral sclerosis: preliminary results. Klin Neuroradiol. 2009;19(2):129-34.

23. Blain $C R$, Williams VC, Johnston $C$, Stanton BR, Ganesalingam J, Jarosz JM, et al. A longitudinal study of diffusion tensor MRI in ALS. Amyotroph Lateral scler. 2007;8(6):348-55.

24. Alruwaili ARP K, Henderson RD, Gray M, Kurniawan ND, McCombe PA. Tract integrity in Amyotrophic lateral sclerosis: 6-month evaluation using MR diffusion tensor imaging. BMC Med Imaging. 2019;19:19.

25. Albuquerque EX, Pereira EF, Alkondon M, Rogers SW. Mammalian nicotinic acetylcholine receptors: from structure to function. Physiol Rev. 2009;89(1):73-120.

26. Menke RAL, Proudfoot M, Talbot K, Turner MR. The two-year progression of structural and functional cerebral MRI in amyotrophic lateral sclerosis. Neurolmage Clin. 2018;17:953-61.

27. Brooks BR, Miller RG, Swash M, Munsat TL. El Escorial revisited: revised criteria for the diagnosis of amyotrophic lateral sclerosis. Amyotroph Lateral Scler Other Motor Neuron Disord. 2000;1(5):293-9.

28. Cedarbaum JM, Stambler N, Malta E, Fuller C, Hilt D, Thurmond B, et al. The ALSFRS-R: a revised ALS functional rating scale that incorporates assessments of respiratory function. BDNF ALS study group (phase III). J Neurol Sci. 1999;169(1-2):13-21.

29. Pannek K, Boyd RN, Fiori S, Guzzetta A, Rose SE. Assessment of the structural brain network reveals altered connectivity in children with unilateral cerebral palsy due to periventricular white matter lesions. Neurolmage: Clin. 2014:5:84-92.

30. Tournier J, Calamante F, Connelly A. MRtrix: diffusion tractography in crossing fiber regions. Int J Imaging Syst Technol. 2012;22(1):53-66.

31. Liu Y, Spulber G, Lehtimaki KK, Kononen M, Hallikainen I, Grohn H, et al. Diffusion tensor imaging and tract-based spatial statistics in Alzheimer's disease and mild cognitive impairment. Neurobiol Aging. 2011;32(9):1558-71.

32. Smith SM, Nichols TE. Threshold-free cluster enhancement: addressing problems of smoothing, threshold dependence and localisation in cluster inference. Neuroimage. 2009;44(1):83-98.

33. Nichols TE, Holmes AP. Nonparametric permutation tests for functional neuroimaging: a primer with examples. Hum Brain Mapp. 2002;15(1):1-25.

34. Avants BB, Tustison N, Song G. Advanced normalization tools (ANTS). Insight J. 2009;2:1-35

35. Wakana S, Jiang H, Nagae-Poetscher LM, Van Zijl PC, Mori S. Fiber tract-based atlas of human white matter anatomy 1. Radiology. 2004;230(1):77-87.

36. Wakana $\mathrm{S}$, Jiang $\mathrm{H}$, Nagae-Poetscher LM, PCMv Z, Mori S. Fiber tract-based atlas of human white matter anatomy. Radiology. 2004;230(1):77-87.

37. Koch GG. Intraclass correlation coefficient, Encyclopedia of Statistical Sciences: Wiley; 2004. https://doi.org/10.1002/9781118445112.stat02461.

38. Bonekamp D, Nagae-Poetscher L, Degaonkar M, Matson M, Mori S, Horska A, editors. Intra-rater and inter-rater reproducibility of FA and ADC: a clinical pediatric DTI study. Proceedings of the 13th ISMRM Scientific Meeting, Miami, Florida; 2005.

39. Bede P, Querin G, Pradat P-F. The changing landscape of motor neuron disease imaging: the transition from descriptive studies to precision clinical tools. Curr Opin Neurol. 2018;31(4):431-8.

40. Jacob S, Finsterbusch J, Weishaupt JH, Khorram-Sefat D, Frahm J, Ehrenreich $\mathrm{H}$. Diffusion tensor imaging for long-term follow-up of corticospinal tract degeneration in amyotrophic lateral sclerosis. Neuroradiology. 2003:45(9):598-600.
41. Kasper E, Schuster C, Machts J, Kaufmann J, Bittner D, Vielhaber S, et al. Microstructural white matter changes underlying cognitive and behavioural impairment in ALS--an in vivo study using DTI. PLoS One. 2014;9(12):e114543.

42. Mateen FJ, Carone M, Sorenson EJ. Patients who survive 5 years or more with ALS in Olmsted County, 1925-2004. J Neurol Neurosurg Psychiatry. 2010;81(10):1144-6.

43. Turner MR, Parton MJ, Shaw CE, Leigh PN, Al-Chalabi A. Prolonged survival in motor neuron disease: a descriptive study of the King's database 19902002. J Neurol Neurosurg Psychiatry. 2003;74(7):995-7.

44. Pupillo $E$, Messina $P$, Logroscino $G$, Beghi E. Long-term survival in amyotrophic lateral sclerosis: a population-based study. Ann Neurol. 2014; 75(2):287-97.

45. Menke RA, Korner S, Filippini N, Douaud G, Knight S, Talbot K, et al. Widespread grey matter pathology dominates the longitudinal cerebral MRI and clinical landscape of amyotrophic lateral sclerosis. Brain. 2014;137(Pt 9):2546-55.

46. Devine MS, Kiernan MC, Heggie S, McCombe PA, Henderson RD. Study of motor asymmetry in ALS indicates an effect of limb dominance on onset and spread of weakness, and an important role for upper motor neurons. Amyotroph Lateral Scler Frontotemporal Degener. 2014;15(7-8):481-7.

47. Vucic S, Cheah BC, Kiernan MC. Maladaptation of cortical circuits underlies fatigue and weakness in ALS. Amyotroph Lateral Scler. 2011;12(6):414-20.

48. Kassubek J, Muller HP, Del Tredici K, Brettschneider J, Pinkhardt EH, Lule D, et al. Diffusion tensor imaging analysis of sequential spreading of disease in amyotrophic lateral sclerosis confirms patterns of TDP-43 pathology. Brain. 2014;137(Pt 6):1733-40.

49. Prokscha T, Guo J, Hirsch S, Braun J, Sack I, Meyer T, et al. Diffusion tensor imaging in amyotrophic lateral sclerosis--increased sensitivity with optimized region-of-interest delineation. Clin Neuroradiol. 2014;24(1):37-42.

50. Mori S, Oishi K, Jiang H, Jiang L, Li X, Akhter K, et al. Stereotaxic white matter atlas based on diffusion tensor imaging in an ICBM template. Neurolmage. 2008;40(2):570-82.

51. Oishi K, Faria A, Jiang H, Li X, Akhter K, Zhang J, et al. Atlas-based whole brain white matter analysis using large deformation diffeomorphic metric mapping: application to normal elderly and Alzheimer's disease participants. Neurolmage. 2009;46(2):486-99.

52. Babalola KO, Patenaude B, Aljabar P, Schnabel J, Kennedy D, Crum W, et al. An evaluation of four automatic methods of segmenting the subcortical structures in the brain. Neurolmage. 2009;47(4):1435-47.

53. Morey RA, Selgrade ES, Wagner HR 2nd, Huettel SA, Wang L, McCarthy G. Scan-rescan reliability of subcortical brain volumes derived from automated segmentation. Hum Brain Mapp. 2010;31(11):1751-62.

54. Aribisala BS, Cox SR, Ferguson KJ, MacPherson SE, MacLullich AM, Royle NA, et al. Assessing the performance of atlas-based prefrontal brain parcellation in an aging cohort. J Comput Assist Tomogr. 2013;37(2):257-64.

55. Zhu T, Hu R, Qiu X, Taylor M, Tso Y, Yiannoutsos C, et al. Quantification of accuracy and precision of multi-center DTI measurements: a diffusion phantom and human brain study. Neuroimage. 2011:56(3):1398-411.

56. Magnotta VA, Matsui JT, Liu D, Johnson HJ, Long JD, Bolster BD Jr, et al. Multicenter reliability of diffusion tensor imaging. Brain Connectivity. 2012; 2(6):345-55.

57. Bonekamp D, Nagae LM, Degaonkar M, Matson M, Abdalla WM, Barker PB, et al. Diffusion tensor imaging in children and adolescents: reproducibility, hemispheric, and age-related differences. Neuroimage. 2007;34(2):733-42.

\section{Publisher's Note}

Springer Nature remains neutral with regard to jurisdictional claims in published maps and institutional affiliations. 